\section{The First Total Synthesis of Pyralomicin 1c}

Sir:

Pyralomicins $1 \mathrm{c}$ and $2 \mathrm{c}$ ( $\mathbf{1}$ and 2 ) have been isolated from culture broth of Microtetraspora spiralis as novel antibiotics including antitumor activities ${ }^{1)}$. Sturcturally, 1 and 2 are endowed with the 5-hydroxy-8-methyl-[1]benzopyrano[2,3-b]pyrrol-4-(1H)-one structure as a common core binding a carba sugar and a sugar moiety, respectively $^{2,3)}$ (Fig. 1).

Recently, pyralomicin 2c (2) was synthesized in our laboratories ${ }^{4)}$ from the aglycone, pyralomicinone (5) and properly protected D-glucose 6 (Fig. 2).

Herein, we describe the total synthesis of pyralomicin $1 \mathrm{c}$ (1) to confirm the absolute structure.

Since pyralomicinone (5) has been synthesized from pyrrole and 2,4-dihydroxytoluene derivatives (for examples: 3 and $\mathbf{4})^{4,5)}$, the first aim in the present synthesis is the effective construction of the carba sugar moiety $\mathbf{2 3}$.

We expected the regio- and stereoselective connection of 23 with pyralomicinone (5) to be controlled under Mitsunobu conditions ${ }^{4)}$ with inversion.

Furthermore, it was anticipated that the carba sugar $\mathbf{2 3}$ would be synthesized by the similar strategies as developed by $\mathrm{us}^{6,7)}$ in the synthesis of glyoxalase I inhibitor ${ }^{8)}(13)$ and its precursor, KD16-U1 ${ }^{9)}$ (12) (Fig. 3). Both carbocycles 12 and 13 have been synthesized from D-ribonic acid $\gamma$-lactone 7 through one-step opening of the furanose 8 followed by aldol condensation $(\mathbf{9} \sim \mathbf{1 1})$.

The starting material we came to favor was L-arabinonic acid $\gamma$-lactone 14, which was readily derived from Larabinose by tritylation and bromine oxidation ${ }^{10)}$ (Fig. 4).

Silylation of 14 followed by de- $O$-tritylation gave the alcohol 15, which was submitted to Pfitzner-Moffatt oxidation and acetal formation to afford the acetal 16 (Table 1). Reaction of $\mathbf{1 6}$ with lithiated methyl phenyl sulfonate gave the furanose $\mathbf{1 7}$ in a quantitative yield. This was silylated to the opened chain 18 in one step by simultaneous formation of an enol silyl ether and an $O$-silyl secondary alcohol as mentioned before ${ }^{6,7)}$.

Fig. 1 .<smiles></smiles>

1: Pyralomicin 1c<smiles></smiles>

2: Pyralomicin $2 \mathrm{c}$

Fig. 2.

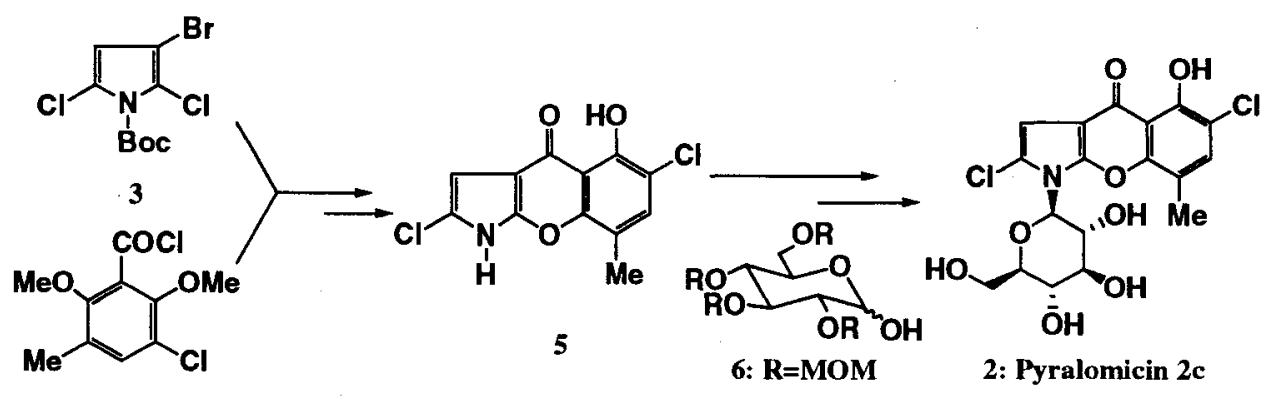


Fig. 3.

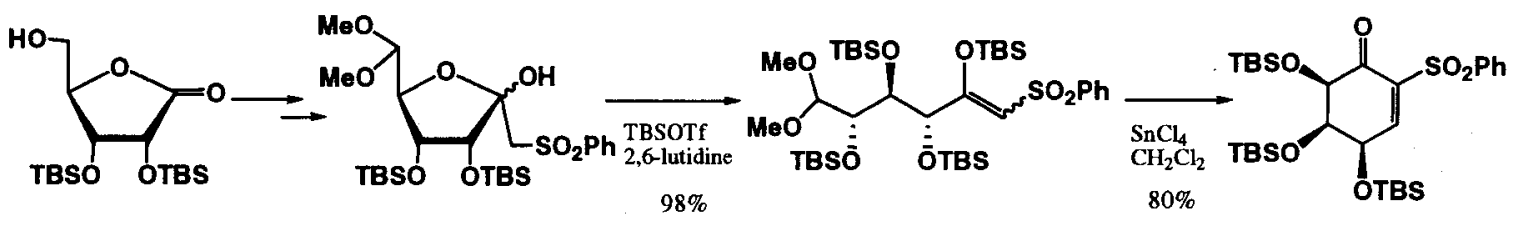

7

8

9

10

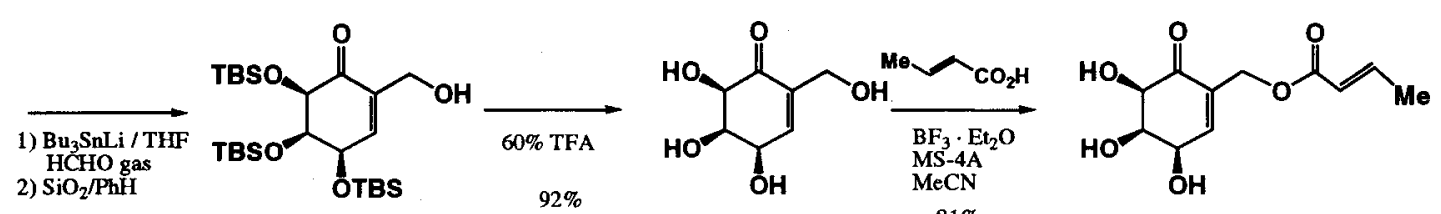
$88 \%$

Fig. 4.

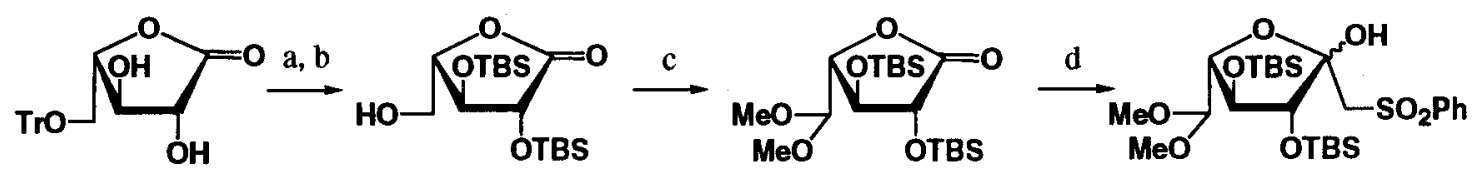
14 15 16 17

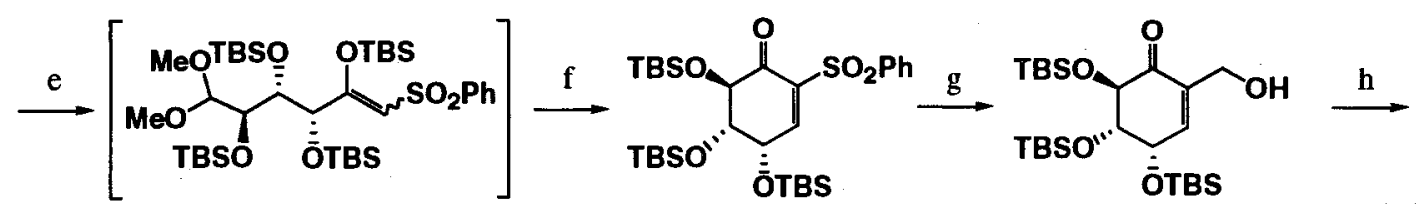
18 19 20

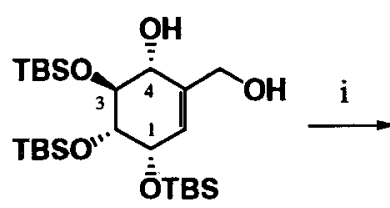

21<smiles></smiles>

22

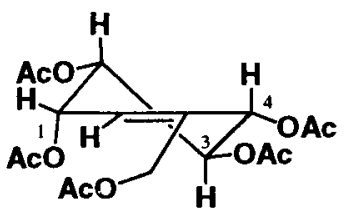<smiles>COCC1=C[C@H](O)[C@@H](O)[C@H](O)[C@H]1OC</smiles>

23

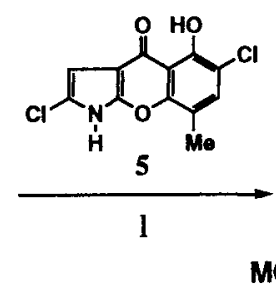

MOMO<smiles>CO[C@@H]1C(CO)=C[C@@H](n2c(Cl)cc3c(=O)c4c(O)c(Cl)cc(C)c4oc32)[C@H](O)[C@@H]1O</smiles>

24<smiles>Cc1cc(Cl)c(O)c2c(=O)c3cc(Cl)n([C@@H]4C=C(CO)[C@@H](O)[C@H](O)[C@H]4O)c3oc12</smiles>

1: Pyralomicin 1c

Conditions; (a) TBSOTf, 2,6-lutidine/ $\mathrm{CH}_{2} \mathrm{Cl}_{2}, \mathrm{rt}, 3$ hours; 94\% (b) $\mathrm{H}_{2}, \mathrm{Pd}-\mathrm{C} / \mathrm{CHCl}_{3}, \mathrm{rt}, 12$ hours; 85\% (c) 1) DCC, Py॰TFA, DMSO/Et ${ }_{2} \mathrm{O}, \mathrm{rt}, 3$ hours 2) CSA, $\mathrm{HC}(\mathrm{OMe})_{3} / \mathrm{MeOH}, 50^{\circ} \mathrm{C}, 36$ hours; $81 \%$ (d) $\mathrm{MeSO}_{2} \mathrm{Ph}, n$-BuLi/THF, $-78^{\circ} \mathrm{C}$, 1.5 hours; $97 \%$ (e) TBSOTf, 2,6 -lutidine/ $\mathrm{CH}_{2} \mathrm{Cl}_{2}, 40^{\circ} \mathrm{C}, 24$ hours; $94 \%$ (f) $\mathrm{SnCl}_{4} / \mathrm{CH}_{2} \mathrm{Cl}_{2},-78^{\circ} \mathrm{C}, 15$ minutes; $71 \%$ (g) $n$-Bu $\mathrm{Bu}_{3} \mathrm{SnLi}, \mathrm{HCHO} / \mathrm{THF},-78^{\circ} \mathrm{C}$ to $40^{\circ} \mathrm{C}, 24$ hours; $74 \%$

(h) $\mathrm{NaBH}_{4}, \mathrm{CeCl}_{3} \cdot 7 \mathrm{H}_{2} \mathrm{O} / \mathrm{MeOH}-\mathrm{CH}_{2} \mathrm{Cl}_{2},-78^{\circ} \mathrm{C}, 3.5$ hours; $69 \%$ (i) 1) TBAF/THF, rt, 4 hours 2) $\mathrm{Ac}_{2} \mathrm{O}$, Py, rt, 12 hours; $94 \%$ (j) $\mathrm{MOMCl}, n-\mathrm{Bu}_{4} \mathrm{NI}$, DIPEA$/ \mathrm{CH}_{2} \mathrm{Cl}_{2}, 50^{\circ} \mathrm{C}, 24$ hours; $92 \%$ (k) TBAF/THF, rt, 4 hours; $95 \%$ (l) $n$-Bu $3 \mathrm{P}=\mathrm{CHCN} / \mathrm{THF}, 40^{\circ} \mathrm{C}, 12$ hours; $51 \%$ (m) $5 \% \mathrm{HCl} / \mathrm{MeOH}, 50^{\circ} \mathrm{C}, 4$ hours; $97 \%$ 
Table 1. Physico-chemical properties of compounds.

\begin{tabular}{|c|c|c|c|}
\hline No. & $\mathrm{Mp}\left({ }^{\circ} \mathrm{C}\right)$ & $\begin{array}{c}{[\alpha]_{D}} \\
\left(\mathrm{CHCl}_{3}\right)\end{array}$ & ${ }^{1} \mathrm{H}-\mathrm{NMR}\left(270,300\right.$ or $\left.600 \mathrm{MHz} ; \mathrm{CDCl}_{3} ; \delta \mathrm{ppm} ; J \mathrm{~Hz}\right)$ \\
\hline 1 & $\begin{array}{l}\text { 283-286 } \\
\text { (decomp.) }\end{array}$ & $\begin{array}{c}-148^{\circ} \\
\text { (c } 0.24, \mathrm{DMF})\end{array}$ & $\begin{array}{l}\left(\mathrm{DMF}-d_{7}\right): \delta 2.34(3 \mathrm{H}, \mathrm{s}), 3.70(1 \mathrm{H}, \mathrm{ddd}, J=5.0,8.0 \& 10.2) \\
4.18-4.40(4 \mathrm{H}, \mathrm{m}), 4.86-4.92(1 \mathrm{H}, \mathrm{m}), 5.14(1 \mathrm{H}, \mathrm{br} \mathrm{s}), 5.28(1 \mathrm{H}, \mathrm{d} \\
J=5.0), 5.32(1 \mathrm{H}, \mathrm{br} \mathrm{d}, J=6.2), 5.58(1 \mathrm{H}, \text { br s }), 5.72(1 \mathrm{H}, \mathrm{br} \mathrm{s}) \\
6.72(1 \mathrm{H}, \mathrm{s}), 7.70(1 \mathrm{H}, \mathrm{s}), 13.82(1 \mathrm{H}, \mathrm{br} \mathrm{s})\end{array}$ \\
\hline 14 & Foam & $\begin{array}{c}+17^{\circ} \\
(c \quad 1.18)\end{array}$ & $\begin{array}{l}\delta 3.37(1 \mathrm{H}, \mathrm{dd}, J=4.0 \& 10.4), 3.54(1 \mathrm{H}, \mathrm{dd}, J=3.8 \& 10.4), 4.25(1 \mathrm{H}, \\
\mathrm{ddd}, J=3.8,4.0 \& 8.2), 4.36(1 \mathrm{H}, \mathrm{dd}, J=8.2 \& 8.2), 4.45(1 \mathrm{H}, \mathrm{d}, \\
J=8.2), 7.20-7.46(15 \mathrm{H}, \mathrm{m})\end{array}$ \\
\hline 15 & Foam & $\begin{array}{c}-5.6^{\circ} \\
(c \quad 1.15)\end{array}$ & $\begin{array}{l}\delta 0.13(3 \mathrm{H}, \mathrm{s}), 0.14(3 \mathrm{H}, \mathrm{s}), 0.15(3 \mathrm{H}, \mathrm{s}), 0.21(3 \mathrm{H}, \mathrm{s}), 0.90(9 \mathrm{H}, \mathrm{s}) \\
0.94(9 \mathrm{H}, \mathrm{s}), 1.94(1 \mathrm{H}, \mathrm{br} \mathrm{s}), 3.73(1 \mathrm{H}, \mathrm{ddd}, J=3.8,8.0 \& 14.0) \\
3.95(1 \mathrm{H}, \mathrm{ddd}, J=2.2,6.0 \& 14.0), 4.11(1 \mathrm{H}, \mathrm{ddd}, J=2.2,3.8 \& 7.0) \\
4.33(1 \mathrm{H}, \mathrm{dd}, J=7.0 \& 7.0), 4.38(1 \mathrm{H}, \mathrm{d}, J=7.0)\end{array}$ \\
\hline 16 & Syrup & $\begin{array}{c}+16^{\circ} \\
(c \quad 1.28)\end{array}$ & $\begin{array}{l}\delta 0.10(3 \mathrm{H}, \mathrm{s}), 0.11(3 \mathrm{H}, \mathrm{s}), 0.16(3 \mathrm{H}, \mathrm{s}), 0.19(3 \mathrm{H}, \mathrm{s}), 0.88(9 \mathrm{H}, \mathrm{s}) \\
0.92(9 \mathrm{H}, \mathrm{s}), 3.44(3 \mathrm{H}, \mathrm{s}), 3.45(3 \mathrm{H}, \mathrm{s}), 4.17(1 \mathrm{H}, \mathrm{dd}, J=4.6 \& 5.0), \\
4.19(1 \mathrm{H}, \mathrm{d}, J=4.6), 4.26(1 \mathrm{H}, \mathrm{dd}, J=4.6 \& 4.6), 4.47(1 \mathrm{H}, \mathrm{d}, J=5.0)\end{array}$ \\
\hline 19 & $131-132$ & $\begin{array}{c}+87^{\circ} \\
(c \quad 0.74)\end{array}$ & $\begin{array}{l}\delta-0.08(3 \mathrm{H}, \mathrm{s}),-0.06(3 \mathrm{H}, \mathrm{s}),-0.02(3 \mathrm{H}, \mathrm{s}), 0.05(3 \mathrm{H}, \mathrm{s}), 0.15(3 \mathrm{H}, \\
\mathrm{s}), 0.17(3 \mathrm{H}, \mathrm{s}), 0.57(9 \mathrm{H}, \mathrm{s}), 0.81(9 \mathrm{H}, \mathrm{s}), 0.95(9 \mathrm{H}, \mathrm{s}), 3.81(1 \mathrm{H}, \mathrm{d}, \\
J=5.0), 4.01(1 \mathrm{H}, \mathrm{ddd}, J=2.0,2.0 \& 5.0), 5.10(1 \mathrm{H}, \mathrm{dd}, J=1.0 \& 2.0) \\
7.49(3 \mathrm{H}, \mathrm{m}), 7.74(1 \mathrm{H}, \mathrm{dd}, J=1.0 \& 2.0), 7.94-8.00(2 \mathrm{H}, \mathrm{m})\end{array}$ \\
\hline 20 & Syrup & $\begin{array}{c}+73^{\circ} \\
(c \quad 1.20)\end{array}$ & $\begin{array}{l}\delta 0.04(3 \mathrm{H}, \mathrm{s}), 0.05(3 \mathrm{H}, \mathrm{s}), 0.06(3 \mathrm{H}, \mathrm{s}), 0.12(3 \mathrm{H}, \mathrm{s}), 0.13(6 \mathrm{H}, \mathrm{s}), \\
0.81(9 \mathrm{H}, \mathrm{s}), 0.87(9 \mathrm{H}, \mathrm{s}), 0.93(9 \mathrm{H}, \mathrm{s}), 2.22(1 \mathrm{H}, \mathrm{t}, J=7.0) \\
3.83-4.06(2 \mathrm{H}, \mathrm{m}), 4.19(1 \mathrm{H}, \mathrm{dd}, J=6.0 \& 14.0), 4.31(1 \mathrm{H}, \mathrm{dd}, \\
J=5.0 \& 14.0), 4.85-4.95(1 \mathrm{H}, \mathrm{m}), 6.51(1 \mathrm{H}, \mathrm{br} \mathrm{s})\end{array}$ \\
\hline 21 & Syrup & $\begin{array}{c}+35^{\circ} \\
(c 2.18)\end{array}$ & $\begin{array}{l}\delta 0.08(3 \mathrm{H}, \mathrm{s}), 0.09(3 \mathrm{Hx} 3, \mathrm{~s}), 0.12(3 \mathrm{H}, \mathrm{s}), 0.13(3 \mathrm{H}, \mathrm{s}), 0.85(9 \mathrm{H}, \\
\mathrm{s}), 0.87(9 \mathrm{H}, \mathrm{s}), 0.92(9 \mathrm{H}, \mathrm{s}), 2.17(1 \mathrm{H}, \mathrm{br} \mathrm{s}), 3.17(1 \mathrm{H}, \mathrm{d}, J=12.0) \\
3.75(1 \mathrm{H}, \mathrm{d}, J=12.0), 3.88-3.93(1 \mathrm{H}, \mathrm{m}), 4.04(1 \mathrm{H}, \mathrm{dd}, J=2.0 \& 4.2) \\
4.15-4.30(2 \mathrm{H}, \mathrm{m}), 4.59(1 \mathrm{H}, \mathrm{dd}, J=2.0 \& 2.0), 5.52(1 \mathrm{H}, \mathrm{dd} \\
J=2.0 \& 2.0)\end{array}$ \\
\hline 22 & Syrup & $\begin{array}{c}+51^{\circ} \\
\text { (c } 0.54)\end{array}$ & $\begin{array}{l}\delta 2.02(3 \mathrm{H}, \mathrm{s}), 2.04(3 \mathrm{H}, \mathrm{s}), 2.06(3 \mathrm{H}, \mathrm{s}), 2.07(3 \mathrm{H}, \mathrm{s}), 2.11(3 \mathrm{H}, \mathrm{s}) \\
4.41(1 \mathrm{H}, \mathrm{d}, J=13.0), 4.71(1 \mathrm{H}, \mathrm{d}, J=13.0), 5.16(1 \mathrm{H}, \mathrm{dd} \\
J=4.0 \& 10.4), 5.53(1 \mathrm{H}, \mathrm{dd}, J=7.6 \& 10.4), 5.62(1 \mathrm{H}, \mathrm{dd} \\
J=4.0 \& 5.8), 5.70(1 \mathrm{H}, \mathrm{d}, J=7.6), 5.97(1 \mathrm{H}, \mathrm{d}, J=5.8)\end{array}$ \\
\hline 23 & $111-113$ & $\begin{array}{c}+5.3^{\circ} \\
(c \quad 2.18)\end{array}$ & $\begin{array}{l}\left(\mathrm{CD}_{3} \mathrm{OD}\right): \delta 3.30(3 \mathrm{H}, \mathrm{s}), 3.39(3 \mathrm{H}, \mathrm{s}), 3.40(1 \mathrm{H}, \mathrm{dd}, \\
J=4.2 \& 10.0), 3.80(1 \mathrm{H}, \mathrm{dd}, J=7.6 \& 10.0), 3.92(1 \mathrm{H}, \mathrm{dd}, \\
J=1.0 \& 7.6), 4.03(1 \mathrm{H}, \mathrm{d}, J=12.4), 4.11-4.15(2 \mathrm{H}, \mathrm{m}), 4.56(1 \mathrm{H}, \mathrm{d}, \\
J=6.0), 4.59(1 \mathrm{H}, \mathrm{d}, J=6.0), 4.71(1 \mathrm{H}, \mathrm{d}, J=6.0), 4.88(1 \mathrm{H}, \mathrm{d}, \\
J=6.0), 5.88(1 \mathrm{H}, \mathrm{dd}, J=2.0 \& 5.0)\end{array}$ \\
\hline 24 & $214-216$ & $\begin{array}{c}-104^{\circ} \\
(c \quad 0.31)\end{array}$ & $\begin{array}{l}\delta 2.28(3 \mathrm{H}, \mathrm{s}), 3.30(3 \mathrm{H}, \mathrm{s}), 3.53(3 \mathrm{H}, \mathrm{s}), 3.74(1 \mathrm{H}, \mathrm{d}, J=2.4), \\
3.86(1 \mathrm{H}, \mathrm{dd}, J=7.8 \& 8.2), 4.12-4.24(3 \mathrm{H}, \mathrm{m}), 4.38(1 \mathrm{H}, \mathrm{ddd}, J=2.4 \text {, } \\
8.2 \& 8.2), 4.60(1 \mathrm{H}, \mathrm{d}, J=5.8), 4.62(1 \mathrm{H}, \mathrm{d}, J=5.8), 4.74(1 \mathrm{H}, \mathrm{br} s) \\
4.84(1 \mathrm{H}, \mathrm{d}, J=7.0), 4.91(1 \mathrm{H}, \mathrm{d}, J=7.0), 5.20(1 \mathrm{H}, \mathrm{br} \mathrm{d}, J=2.4), \\
5.77(1 \mathrm{H}, \mathrm{br} \mathrm{s}), 6.49(1 \mathrm{H}, \mathrm{s}), 7.37(1 \mathrm{H}, \mathrm{s}), 13.29(1 \mathrm{H}, \mathrm{s})\end{array}$ \\
\hline
\end{tabular}

The $\mathrm{SnCl}_{4}$-promoted aldol condensation of the 18 resulted in the formation of the cyclohexenone 19, which was treated with tributylstannyl lithium ${ }^{11)}$ followed by reation with formaldehyde to yield the $\alpha$-hydroxymethylcyclohexenone 20 with elimination of $\mathrm{Bu}_{3} \mathrm{Sn}$ and $\mathrm{PhSO}_{2}$ groups.

Stereoselective reduction of the carbonyl group of $\mathbf{2 0}$ was assayed in a variety of conditions, and the best result was realized by using $\mathrm{NaBH}_{4}$ and $\mathrm{CeCl}_{3} \cdot 7 \mathrm{H}_{2} \mathrm{O}$ to give the desired $\alpha$-alcohol 21 in $69 \%$ yield with $10 \%$ of the $\beta$ - 
alcohol. The configuration of the newly produced $\mathrm{C}-4$ hydroxy group was not determined by the ${ }^{1} \mathrm{H}-\mathrm{NMR}$ studies of 21, while the configuration was unambiguously disclosed by those of the corresponding penta- $O$-acetate 22 , which reasonably presented in a half-chair conformation ${ }^{12)}$ as shown in Fig. 4: $J_{1,2}=4.0 \mathrm{~Hz}, J_{2,3}=10.4 \mathrm{~Hz}, J_{3,4}=7.6 \mathrm{~Hz}$, and $J_{1,6}=5.8 \mathrm{~Hz}$.

The alcohol 21 was protected with methoxymethyl group followed by de- $O$-silylation to give quantitatively the triol 23. Although 23 possessed three free hydroxy groups, the allyl hydroxy group at $\mathrm{C}-1$ was expected to be more reactive than others.

With pyralomicinone (5) and the alcohol 23 in hand, we turned to their connection.

Both components 5 and $\mathbf{2 3}$ were coupled under modified Mitsunobu's conditions ${ }^{13)}$ using a novel reagent, $n$ $\mathrm{Bu}_{3} \mathrm{P}=\mathrm{CHCN}$ to give predominanlty the desired product $\mathbf{2 4}$ with inversion. As expected, the by-products which would result from the reaction of other hydroxy groups with 5 were not significantly observed.

Acidic deprotection of 24 produced pyralomicin 1c (1), which was identical with the natural product ${ }^{1-3)}$ in all respects, completing the first total synthesis.

Now that the synthesis of pyralomicins $1 \mathrm{c}(\mathbf{1})$ and $2 \mathrm{c}(\mathbf{2})$ including a carba sugar and a sugar moiety, respectively, have been accomplished, the synthesis of other families ${ }^{1-3)}$ is the subject of current studies.

\section{Acknowledgment}

We are grateful to Meiji Seika Kaisha, Ltd., Advanced Research Institute for Science and Engineering, Waseda University, and High-Tech Research Center Project the Ministry of Education, Science, Sports and Culture for the generous support of our program. The present work was financially supported by Grant-in-Aid for Specially Promoted Research from the Ministry of Education, Science, Sports and Culture. We also thank Ms. TOMOKO WATANABE for her experimental assistance, and Dr. H. NAGANAWA, Institute of Microbial Chemistry, for kindly providing the natural products.

\section{KUNIAKI TATSUTA* \\ MASAAKI TAKAHASHI NobOrU TANAKA}

Department of Applied Chemistry, School of Science and Engineering, Waseda University, 3-4-1 Ohkubo, Shinjuku, Tokyo 169-8555, Japan

\section{References}

1) KaWamura, N.; N. Kinoshita, R. SaWa, Y. TAKahashi, T. SaWA, H. Naganawa, M. Hamada \& T. Takeuchi: Pyralomicins, novel antibiotics from Microtetraspora spiralis. I. Taxonomy and production. J. Antibiotics 49: 706 709, 1996

2) Kawamura, N.; R. Sawa, Y. Takahashi, K. Isshiki, T. SaWa, H. Naganawa \& T. Takeuchi: Pyralomicins, novel antibiotics from Microtetraspora spiralis. II. Structure determination. J. Antibiotics 49: 651 656, 1996

3) KaWamura, N.; H. Nakamura, R. SaWa, Y. Takahashi, T. SAWA, H. NAGanaWA \& T. TAKeUChI: Pyralomicins, novel antibiotics from Microtetraspora spiralis. IV. Absolute configuration. J. Antibiotics 50: 147 149, 1997

4) Tatsuta, K.; M. Takahashi \& N. Tanaka: The first total synthesis of pyralomicin 2c. Tetrahedron Lett. 40: 1929 1932, 1999

5) Kelly, T. R. \& R. L. Moiseyeva: Total synthesis of the pyralomicinones. J. Org. Chem. 63: 3147 3150, 1998

6) Tatsuta, K.; S. Yasuda, K. Kurihara, K. Tanabe, R. SHINEI \& T. OKONOGI: Total synthesis of progesterone receptor ligands, (-)-PF1092A, B and C. Tetrahedron Lett. 38: 1439 1442, 1997

7) Tatsuta, K.; S. Yasuda, N. Araki, M. Takahashi \& Y. KAMIYA: Total synthesis of a glyoxalase I inhibitor and its precusor, (-)-KD16-U1. Tetrahedron Lett. 39: 401 402, 1998

8) Takeuchi, T.; H. Chimura, M. Hamada, H. Umezawa, O. Yoshioka, N. Oguchi, Y. TAKahashi \& A. Matsud́a: The glyoxalase I inhibitor of a new structural type produced by Streptomyces. J. Antibiotics 28: 737 742, 1975

9) Tatsuta, K.; T. Tsuchiya, N. Mikami, S. Umezawa, H. UMEZAWA \& H. NAGANAWA: KD16-U1, a new metabolite of Streptomyces. Isolation and structural studies. J. Antibiotics 27: 579 586, 1974

10) Overend, W. G.; M. Stacey \& L. F. Wrggins: Deoxysugars. part IV. A synthesis of 2-deoxy-D-ribose from Derythrose. J. Chem. Soc. 1949: 1358 1363, 1949

11) OchiaI, M.; T. UkiTA \& E. FuJita: A new desulphonylation of $\alpha, \beta$-unsaturated sulphones via conjugate addition of tributylstannyl-lithium. J. Chem. Soc. Chem. Commun. 1983: 619 620, 1983

12) Abraham, R. J.; H. Gottschalck, H. Paulsen \& W. A. THomas: The proton magnetic resonance spectra and conformations of cyclic compounds. part II. The p.m.r. spectra of the conduritols. J. Chem. Soc. 1965: 6268 6277,1965

13) Tsunoda, T.; F. Ozaki, N. Shirakata, Y. Tamaoka, H. YAMAMOTO \& S. ITO: Formation of heterocycles by the Mitsunobu reaction. Stereoserective synthesis of $(+)-\alpha-$ skytanthine. Tetrahedron Lett. 37: 2463 2466, 1996 